CLINICAL STUDY

\title{
Diagnostic and prognostic value of immunocytochemistry and BRAF mutation analysis on liquid-based biopsies of thyroid neoplasms suspicious for carcinoma
}

\author{
Esther Diana Rossi ${ }^{1}$, Maurizio Martini ${ }^{1}$, Sara Capodimonti ${ }^{1}$, Patrizia Straccia ${ }^{1}$, Tonia Cenci ${ }^{1}$, \\ Celestino Pio Lombardi ${ }^{2}$, Alfredo Pontecorvi ${ }^{3}$, Luigi Maria Larocca ${ }^{1,{ }^{*}}$ and Guido Fadda ${ }^{1,{ }^{*}}$ \\ ${ }^{1}$ Division of Anatomic Pathology and Histology, ${ }^{2}$ Division of Endocrine Surgery and ${ }^{3}$ Division of Endocrinology, Agostino Gemelli' School of Medicine, \\ Università Cattolica del Sacro Cuore, Largo Francesco Vito 1, 00168 Rome, Italy
}

(Correspondence should be addressed to E D Rossi; Email: esther.rossi@rm.unicatt.it)

*(L M Larocca and G Fadda shared senior authorship)

\begin{abstract}
Objective: In the field of fine-needle aspiration cytology, the category of suspicious for malignancy (SM) thyroid lesions, that bears $55-85 \%$ risk of malignant histology, is a challenging topic in which morphology alone is not always able to make a correct diagnosis. Recently, immunocytochemistry (ICC) has been referred to as helpful in differentiating low- and high-malignant risk lesions and BRAF activating mutations have been identified in a significant amount of papillary thyroid carcinomas (PTC). The introduction of the liquid-based cytology (LBC) may simplify the application of these techniques to thyroid cytology.

Design: Our aim is to evaluate the diagnostic and prognostic role of both ICC and BRAF mutation for the SM category on LBC.

Methods: From October 2010 through June 2011, 113 LBC cytological cases (including 37 SM and 76 PTC) underwent surgery. All cases were studied for BRAF mutation and ICC.

Results: ICC resulted positive in $26(86.6 \%)$ histologically malignant SM with 15 of which $(40.5 \%)$ expressing a $B R A F$ mutation. Overall, 63 cases showed a $B R A F$ mutation resulting in PTC. Concerning the prognostic role of $B R A F$ mutation for the two categories, we reported a significant correlation with multifocality, nodal involvement and extra-capsular invasion $(P<0.0001)$.

Conclusions: Special techniques such as ICC and molecular markers might be successfully carried out on LBC-processed material. For both categories, ICC is more sensitive whereas BRAF analysis is an interesting support due to its high specificity adding a prognostic value in both SM and PTCs.
\end{abstract}

European Journal of Endocrinology 168 853-859

\section{Introduction}

Thyroid nodules are a common finding in the general population, taking into account both non-neoplastic and neoplastic entities, representing an area fraught with controversy for which fine-needle aspiration cytology (FNAC) is the most important and generally the first diagnostic tool $(1,2,3)$.

FNAC is a worldwide application because of its simplicity, safety and cost-effectiveness, leading to a correct diagnosis in more than $70 \%$ and to a correct clinical approach in more than $90 \%$ of patients (1).

Nevertheless, the majority of all thyroid FNACs are benign, with $5-10 \%$ being 'malignant' and the remaining $20 \%$ representing a field in which different entities are included. This latter group has been discussed by different international classifications, such as the Bethesda System of Reporting Thyroid
Cytopathology, in which three subcategories have been defined bearing different risks of malignancy and different treatments. On the other hand, the Italian and British cytological classifications encompass only two categories, follicular neoplasm (FN) and suspicious for malignancy (SM), the former including the majority of FN from the Bethesda classification (4).

Apart from these morphological problems, mainly ascribed to the number of false-positive cases, the current recommendation for SM management is reflected in a large consensus for a lobectomy, although in some institutions, a total thyroidectomy is the first initial surgical choice. Looking for an answer, a growing number of experts in the field have increasingly found specific markers (both immunocytochemical and molecular markers) on cytological samples.

The expression of immunohistochemical markers has been proposed as an additional tool for discriminating 
malignant tumours at histology regardless of the presence of capsular and/or vascular invasion. Hector Battifora mesothelial-1 (HBME-1) and Galectin-3 have reached the highest specificity and sensitivity in malignant lesions even if none of the antibodies studied so far have shown a diagnostic accuracy sufficient for its use as single marker of malignancy $(2,3,5,6,7,8)$.

Furthermore, some molecular pathway alterations play a pivotal role in thyroid cancer and, importantly, represent a precocious step in the tumorigenic process justifying their use as uncountable markers of malignancy. The V6OOE BRAF mutation is typically found in $29-69 \%$ of classical papillary thyroid carcinomas (PTC), the most common thyroid malignancy, and in $80 \%$ of the tall cell variant (TCV), while it is less commonly identified in the follicular variant of papillary carcinoma (FVPC) $(9,10,11,12,13)$. The evidence that $B R A F$ mutation could have a diagnostic and prognostic role through progression in a less favourable prognosis has assumed a critical role in the field of cytological thyroid follicular lesions $(10,11,12,14)$.

The application of these special techniques (e.g. molecular markers and immunocytochemistry (ICC)) may show some hitches when applied to conventional cytology. For this reason, in 1996, liquid-based cytology (LBC) has gained popularity as an alternative technique for collection and preparation of cytological specimens based on a methanol-preservative solution and processed with a semi-automated device $(1,2,3,15,16,17,18)$.

It is well-known that there are conflicting opinions and controversial data regarding the efficacy of LBC, although several positive aspects in term of cost-effectiveness, time-sparing, simple application of ancillary techniques such as ICC and molecular biology up to 3-4 months on LBC stored material must be underlined $(3,4,19,20,21,22,23)$.

The aims of this study are i) to evaluate the efficacy and feasibility of LBC for the application of ICC and $B R A F$ molecular analysis to thyroid aspiration cytology and ii) to turn out the diagnostic and prognostic role of these techniques on both SM and malignant categories.

\section{Materials and methods}

From January 2011 through December 2011, a total number of 113 prospective cases (including 37 SM and 76 positive for malignancy) out of 2721 total thyroid FNAB recorded in the files of the Division of Anatomic Pathology and Histology of the Catholic University, 'Agostino Gemelli' Hospital of Rome (Italy), underwent surgery. All 113 cases underwent BRAF analysis and were studied with an immunopanel comprising HBME-1 and Galectin-3. The cases in which the immunopanel results were discordant were further studied for the expression of cytokeratin 19 (CK19). A panel of three antibodies would have been a preferable option but obtaining routinely those additional slides and storing material for molecular analysis in a thyroid FNA often is not possible. All the nodules were evaluated under sonographic guidance (US), mostly by surgeons and endocrinologists, and processed with the LBC method Thin Prep 2000 (Hologic Co., Marlborough, MA, USA). The series included 50 male and 63 female patients with a median age of 42 years (range $20-70$ years). The high M/F rate can probably be ascribed to some bias of our surgical series. All aspirations (usually two passes for each lesion) were performed with $25-27 \mathrm{G}$ needles; no fast assessment of adequacy of the material was done. The nodule sizes ranged from 0.4 to $7 \mathrm{~cm}$. All the subcentimetre lesions were discovered during routine US thyroid check-up performed in the Centre for Thyroid Diseases of the Departments of Endocrinology and Endocrine Surgery of our hospital. All patients had been appropriately informed regarding use of the LBC method for processing their aspiration samples and a written informed consent was signed by all of them.

The aspirated material was fixed with the haemolytic and preservative solution Cytolit after rinsing the needle in this solution. The cells were spun at $50 \boldsymbol{g}$ and then the sediment was transferred into the Preservcyt solution to be processed with the T2000 automated processor according to the manufacturer's suggestions. The resulting slide was fixed in 95\% ethanol and stained with Papanicolaou while the remaining material was stored in the Preservcyt solution at room temperature for 3-4 months to be used for eventual additional investigations. Both ancillary techniques (ICC and BRAF analysis) were applied simultaneously to the cytological diagnosis. These techniques can be performed also when the remaining material is at about $2 \mathrm{ml}$ eluted in $5 \mathrm{ml}$ Preservcyt solution.

The lower limit for the adequacy for each sample was established, according to the British RCPath classification, in six groups of thyroid follicular epithelial cells within the submitted slides, each of them with at least ten well-visualised epithelial cells (24).

The cytological cases were classified according to the Italian Working Group SIAPEC-IAP classification, which shows several overlapping features with both the Bethesda and the British RCPath classifications, especially for the SM category $(4,24,25)$.

The above-mentioned categories are defined as follows: TIR1, inadequate or hemorrhagic; TIR2, nonneoplastic lesion; TIR3, follicular lesion/suspected FN; TIR4, SM and TIR5, positive for malignant neoplasm. Our cytological series presented the following distribution of diagnoses for the reference year: $6.5 \%$ TIR 1 (non-diagnostic), 79\% TIR2 (non-neoplastic), 10.3\% TIR3 (indeterminate), 1.7\% TIR4 (suspicious) and 2.5\% TIR5 (malignant).

The morphological diagnosis of TIR4/SM was achieved in the presence of follicular thyroid cells with nuclear pleomorphisms, irregularities and grooves but without any nuclear inclusion. 


\section{Immunocytochemical analysis}

Immunocytochemical stainings were carried out with the avidin-biotin peroxidase complex on LBC slides using the following antibodies: HBME-1 (Dako, Glostrup, Denmark, 1:100 dilution) and Galectin-3 (Ventana, Tucson, AZ, USA, 1:100 dilution) and CK19 (Dako, 1:100 dilution). The slides were washed three times in PBS and then pre-incubated in normal veal serum with $\mathrm{PBS}$ (1:50) for $20 \mathrm{~min}$ before overnight incubation at $4{ }^{\circ} \mathrm{C}$ with the primary antibody. Then, the slides were washed three times with PBS and incubated with the biotinylated secondary antibody conjugated with the avidin-biotin-peroxidase complex (Ventana). The reaction was developed using 3,3'-diamino-benzidine as a chromogen. All slides were counterstained with haematoxylin for $5 \mathrm{~s}$, rinsed in water three times and then mounted for the microscopic examination. The positivity was assessed, for each cytological case, when at least $50 \%$ of cells showed a strong cytoplasmic positivity. This arbitrary 50\% ICC cut-off was established based on the histological diagnoses. The positivity of each case was defined only when a concomitant positive expression of the two immunomarkers was detected. Positive controls were represented by typical papillary carcinomas and negative controls by histiocytes and lymphocytes identified in most of the thyroid slides.

\section{Histology}

All surgical specimens were fixed in 10\% buffered formaldehyde, embedded in paraffin, and the $5 \mu$-thick microtomic sections were stained with haematoxylineosin. The BRAF mutational analysis was also performed on DNA extracted by surgical specimens, containing at least $70 \%$ of tumour. The concordance of immunohistochemistry and mutational analysis between the surgical and LBC samples was 100\%. All the fibro-adipose tissue close to the thyroid gland was included for the lymph node research.

\section{BRAF mutational analysis}

DNA extraction was performed on fine-needle cytological sample ThinPrep 2000 (Hologic Co.) and paraffin block using the QIAamp tissue kit (Qiagen). The percentage of disease-specific cells for molecular analysis was at least $50 \%$ in all LBC samples and 70\% in surgical specimens. We assessed the quantity and quality of the DNA spectrophotometrically (E260, E260:E280 ratio, spectrum 220-320 nm; Biochrom, Cambridge, UK) and by separation on an Agilent 2100 Bioanalyzer (Palo Alto, CA, USA).

$B R A F$ genes (exons 11 and 15) were amplified using the following primers: for exon 11, forward $5^{\prime}$-TTA TTG ATG CGA ACA GTG AAT AT-3' and reverse 5'-TTA CAG TGG GAC AAA GAA TTG-3'; for exon 15, forward 5'-TCA TAA TGC TTG CTC TGA TAG GA-3' and reverse
5'-GGC CAA AAA TTT AAT CAG TGG A-3'. Briefly, DNA (100-200 ng) was amplified in a mixture containing $1 \times$ PCR buffer $(20 \mathrm{mM}$ Tris $(\mathrm{pH} 8.3), 50 \mathrm{mM} \mathrm{KCl}$ and $1.5 \mathrm{mM} \mathrm{MgCl}_{2}$ ), dNTPs (200 $\mathrm{mM}$ each), primers (20 pM each) and $0.5 \mathrm{U}$ of GoTaq polymerase (Promega) in a final volume of $25 \mu \mathrm{l}$. PCR conditions were as follows: initial denaturation at $95^{\circ} \mathrm{C}$ for $8 \mathrm{~min}$ followed by 35 cycles at $95{ }^{\circ} \mathrm{C}$ for $40 \mathrm{~s}, 55^{\circ} \mathrm{C}$ for $40 \mathrm{~s}$ and $72{ }^{\circ} \mathrm{C}$ for $40 \mathrm{~s}$. After visualisation onto agarose gel, PCR products were treated with ExoSAP-IT (USB Corp., Cleveland, OH, USA) following the manufacturer's protocol, amplified with BigDye Terminator version 3.1 cycle sequencing kit (Applied Biosystems) using forward and reverse primers and sequenced with an ABI PRISM 3100-Avant Genetic Analyzer (Applied Biosystems). Water was used as a negative control.

\section{Statistical analysis}

Statistical analysis was performed using a commercially available statistical software package (SPSS 10.0) for Windows (Microsoft). Comparison of categorical variables was performed by $\chi^{2}$ statistic using the Fisher's exact test when appropriate. A $P$ value $<0.05$ was considered significant.

\section{Results}

One hundred and thirteen FNAC cases processed by LBC underwent surgery. The series includes $37 \mathrm{SM}$ and 76 positive for malignancy/TIR5. All the SMs and malignancies were studied with the application of an immunopanel made up of HBME-1 and Galectin-3. All 113 cases were also evaluated for BRAF mutation. The 76 positive for malignancy/TIR5 were histologically confirmed while 30 out of 37 SM (81\%) were found to be malignant. Table 1 shows the distribution of cytological diagnoses with ICC analysis and BRAF mutation. The ICC analysis, performed in the SM group, was considered informative only in the cases with a concordant positive or negative immunopanel based on the evidence that single immune-marker positivity is not diagnostic alone $(2,5)$. The immunopanel application resulted in 11/37 (30\%) negative SMs and 26/37 (70\%) positive SM cases, with four malignant cases showing positivity only for HBME-1 (Table 1).

Table 1 Cytohistological comparison between BRAF and ICC results.

\begin{tabular}{lccccc}
\hline & FNAC & $\begin{array}{c}\text { Malignant } \\
\text { histology }\end{array}$ & BRAF+ & ICC - & ICC + \\
\hline SM (TIR4) & 37 & 30 & 15 & 11 & 26 \\
PTC (TIR5) & 76 & 76 & 48 & 0 & 76 \\
Overall & 113 & 106 & 63 & 11 & 26 \\
\hline
\end{tabular}

SM, suspicious for malignancy (TIR4, TIR5: positive for malignancy); ICC, immunocytochemistry. 
Table 2 Comparison between ICC and BRAF analysis with the histological diagnosis of SM.

\begin{tabular}{lcccc}
\hline $\begin{array}{c}\text { ICC-, } \\
\text { BRAF }-\end{array}$ & $\begin{array}{r}\text { ICC+, } \\
\text { BRAF+ }\end{array}$ & $\begin{array}{c}\text { ICC-, } \\
\text { BRAF+ }\end{array}$ & $\begin{array}{c}\text { ICC+, } \\
\text { BRAF- }\end{array}$ \\
\hline BL & 7 & 0 & 0 & 0 \\
PC & $1^{\text {a }}$ & 12 & 0 & 5 \\
FVPC & $3^{\text {a }}$ & 1 & 0 & 6 \\
TCV & 0 & 2 & 0 & 0 \\
\hline
\end{tabular}

$\mathrm{BL}$, benign lesion; PC, papillary carcinoma (classic variant); FVPC, follicular variant of PC; TCV, tall cell variant of PC; ICC, immunocytochemistry; SM, suspicious for malignancy (TIR4, TIR5: positive for malignancy).

${ }^{a}$ All these cases showed a discordant panel (two FVPC positive for HBME-1 and cytokeratin 19 whereas one PC and one FVPC only for HBME-1).

In these four discordant cases, we also evaluated that CK19 was positive in two out of the four (50\%). All our papillary carcinomas resulted positive for both immunomarkers and were used as positive control cases. The correlation between histological type and $B R A F$ detection highlighted mutated $B R A F$ in 63 cases out of 113 (48 TIR5 and 15 SM), while 50 out 113 (28 TIR5 and $22 \mathrm{SM}$ ) showed a wild-type sequence. All cases except one (with a double mutation involving two nucleotides at codon 600, GTG $>$ GAA) had a point mutation of GTG to GAG at codon 600 of $B R A F$ exon 15. We did not find any mutation of BRAF exon 11 (Table 1). The sensitivity and specificity of this mutation assay in our laboratory are 85 and $97 \%$ respectively (26). The concordance of mutational analysis between the surgical and LBC samples was $100 \%$.

In Table 2, we compared the results of ICC and $B R A F$ analysis with the histological diagnosis of the SM category, arranging the results in four sub-groups. In detail, the $11 \mathrm{SM}$ with negative immunopanel and BRAF analysis included the seven benign histology and four thyroid cancers (one PTC and three FVPC). All the remaining $26 \mathrm{SMs}$, positive for the immunopanel but with 15 out of $26 \mathrm{BRAF}$-positive detections, were histologically malignant (17 PTC, two TCV and seven FVPC; Table 2).

The data concerning $B R A F$ indicated that all the seven benign lesions were $B R A F$ wild-type and 15 of the 30 malignant cases expressed BRAF mutation. These cases were stratified as 12 classical variants of PTC, two TCV and one in the FVPC subgroup. This result confirms the idea of a more diagnostic role of a positive concordant ICC $(P=0.0007$, OR 55) for the cytological diagnosis of the FVPC histotype (seven cases identified by ICC and just one by BRAF mutation).

In Table 3, we analysed the distribution of histological diagnosis and of parameters of aggressiveness (e.g. multifocality, extra-capsular thyroid invasion and lymph-node metastases) based on the cytological classification. The final histological diagnosis resulted in seven benign follicular adenomas (not specified in that table) and 106 malignant diagnoses including 79 PC (61 in the positive for malignancy/TIR5 group and 18 in SM), 20 FVPC (ten in positive for
malignancy/TIR5 and ten in SM) and seven TCV (five in positive for malignancy/TIR 5 and two in TCV). No false-positive case was found in the positive for malignancy/TIR 5 category. We also analysed the data concerning the three parameters of aggressiveness expressed by 47 extra-capsular thyroid invasion (33 positive for malignancy/TIR 5 and $14 \mathrm{SM}$ ), 41 multifocal cancers ( 24 positive for malignancy/TIR 5 and $17 \mathrm{SM}$ ) and 42 lymph node metastatic disease (27 positive for malignancy/TIR 5 and $15 \mathrm{SM}$ ). All TCV cases were multifocal, with extra-capsular invasion and lymph node involvement (Table 3).

In Table 4, we analysed the relationship between $B R A F$ mutations and parameters of aggressiveness for the two analysed categories of SM and positive for malignancy/TIR 5 .

The presence of a BRAF mutation, for the SM category (Table 4), was significantly linked with evidence of multifocality $(P<0.0001$, OR 0.004 , 95\% CI 0.0002-0.0880), with extra-thyroidal extension $(P<0.0001$, OR 0.025 , 95\% CI $0.0037-0.171)$ and nodal metastases $(P<0.0001$, OR $0.015,95 \% \quad C I$ 0.0019-0.1233).

Concordantly, also in the positive for malignancy/ TIR 5 group (Table 4), the cases with BRAF expression showed a higher bilateral localisation of thyroid cancer $(P=0.0003$, OR 11.00, 95\% CI 2.34-51.65), more frequent lymph node metastases $(P<0.0001$, OR $14.13,95 \%$ CI 3.01-66.3) and extra-capsular infiltration $(P=0.03$, OR $3.26,95 \%$ CI $1.17-9.1)$.

In the SM entity, the sensitivity, specificity, diagnostic accuracy and negative and positive predictive values of immunostainings were 86.6, 100, 89, 63 and 100\% respectively. The same evaluation about $B R A F$ analysis in the same group revealed 50\% sensitivity, $100 \%$ specificity, $60 \%$ diagnostic accuracy and $32 \%$ negative and $100 \%$ positive predictive values respectively.

\section{Discussion}

Although thyroid FNAC represents the most reliable diagnostic approach for establishing the correct diagnosis of either benign or malignant thyroid nodules, a percentage spanning from 10 to $30 \%$ of all cytological samples is included in categories characterised by very

Table 3 Distribution of malignant histological diagnosis and of parameters of aggressiveness based on the cytological classification.

\begin{tabular}{lcccccc}
\hline & PC & FVPC & TCV & $\begin{array}{c}\text { EC } \\
\text { invasion }\end{array}$ & $\begin{array}{c}\text { Multi- } \\
\text { focality }\end{array}$ & $\begin{array}{c}\text { LN } \\
\text { mets }\end{array}$ \\
\hline SM (TIR4) & 18 & 10 & 2 & 14 & 17 & 15 \\
PTC (TIR5) & 61 & 10 & 5 & 33 & 24 & 27 \\
Overall & 79 & 20 & 7 & 47 & 41 & 42
\end{tabular}

PC, papillary carcinoma (classic variant); FVPC, follicular variant of PC; TCV, tall cell variant of PC; ICC, immunocytochemistry; SM, suspicious for malignancy (TIR4, TIR5: positive for malignancy, EC, extra-capsular; LN, lymph node; mets, metastases). 
Table 4 Distribution of multifocality, capsular involvement and nodal metastases based on BRAF molecular pattern in the SM and TIR5 lesions.

\begin{tabular}{lrrc}
\hline & BRAF+ & BRAF- & $\boldsymbol{P}$ \\
\hline SM lesion & & & \\
Unifocal tumour & 0 & 20 & $<0.0001$ \\
Multifocal tumour & 15 & 2 & \\
Intrathyroid tumour & 3 & 20 & $<0.0001$ \\
Extra-thyroid tumour & 12 & 2 & \\
Node negative & 2 & 20 & $<0.0001$ \\
$\quad$ Node positive & 13 & 2 & \\
TIR5 lesion & & & \\
Unifocal tumour & 26 & 26 & 0.0003 \\
Multifocal tumour & 22 & 2 & 0.03 \\
Intrathyroid tumour & 23 & 20 & \\
Extra-thyroid tumour & 25 & 8 & \\
Node negative & 23 & 26 & \\
Node positive & 25 & 2 & \\
\hline
\end{tabular}

different cancer risk (spanning from $5-15$ to $60-75 \%$ ) and variable clinical actions $(4,27,28,29)$.

Even though SM morphology alone may be sufficient for the detection of atypia and nuclear pleomorphisms especially in expert cytological hands, several papers (including a recent one by Mahajan et al. (28)) highlighted the pitfalls of the morphology alone, which can lead to inappropriate treatment (lobectomy vs total thyroidectomy or frozen section), additional morbidity and higher health care costs.

A recent review article by Correia-Rodrigues et al. (30) and other papers have highlighted the increasing application of ancillary techniques to thyroid cytology focusing on immunocytochemical panels as a good choice to discriminate between low- and highmalignant risk lesions that result in an overall diagnostic accuracy spanning from 81 through $92 \%$ with the concordant positive panel $(3,5,29,30,31)$.

The mis-calling SM cases have induced a rising enthusiasm to the possible successful use of ICC and molecular tests on FNAC samples, although many other reports and conferences did not provide any recommendation for ICC in the SM category mainly due to the possibility of false-positive or false-negative immunocytochemical results. In fact, in the present paper, we have found four false-negative malignant cases and recently Cochand-Priollet et al. have found six false-positive LBC thyroid lesions without any falsenegative case when applying an ICC panel of HBME-1 and CK19 $(3,5)$.

One of the drawbacks in routine use of molecular markers on FNAC remains the conventional cytological preparation requiring an adequate quantity of cells and resulting in a difficult scraping of the cells or the need for a second specific aspiration. Our goal, regarding the more detailed technical cytological aspects, was the application of ancillary techniques (both ICC and molecular analyses) on LBC. This method is feasible, highly reproducible and provides high yields with $100 \%$ informative immunocytochemical and molecular results and, furthermore, with a complete concordance between cytology and surgical specimens $(1,2,17,18,22$, $23,30)$.

Our diagnostic everyday approach was reflected in the application of the ICC panel for every SM case achieving a sub-classification in high- and low-risk thyroid lesions (29). This evidence also reflected the present results, where the ICC-positive panel was statistically correlated with a malignant outcome $(P=0.0007)$, highlighted also by the ICC-negative panel in all the seven benign lesions and $86.6 \%$ positive malignancies ( 26 out of 30 cases).

The use of ICC for cytology can suggest a malignant outcome, although the low specificity for these immunomarkers cannot assure a definitive malignant diagnosis. In our four malignant cases with a discordant panel, we added a third immunomarker, specifically CK19, which resulted positive in two out of four, leading to double immunomarker positivity in 50\% of these discordant lesions. We used two immunomarkers for two reasons: i) the limited amount of cytological material and also ii) because in some previous personal experiences, CK19 has also been observed in benign lesions such as lymphocytic thyroiditis and follicular adenomas. To overcome these false-negative results, we looked for the cytological application of BRAF molecular mutational analysis.

The diagnostic utility of the molecular testing panel or single BRAF analysis was strongly encouraged by Nikiforov in several papers, which provided a significant increase in the accuracy of FNAC of up to $95 \%$ $(9,10,14)$. This evidence was also reflected in other authors' experiences, suggesting the use of a scoring model, including cytology and mutational analysis, for classifying correctly $91 \%$ of all samples, with increasing sensitivity from 77 to $96 \%$ and specificity from 68 to $80 \%(32,33)$.

All this rising literature is also reflected in the revised management guidelines for patients with thyroid nodules and differentiated thyroid cancer, recently published by the American Thyroid Association (34), which suggests that the detection of a molecular panel (including BRAF, RAS, RET/PTC and PAX8-PPAR $\gamma$ ) in patients with indeterminate FNA cytology or SM improves the final diagnostic accuracy compared with a single marker.

Furthermore, the detection of BRAF mutation, through the activation of MAPK-kinase pathway, has been correlated with aggressive thyroid tumour features such as extra-thyroidal extension, advanced tumour stage at presentation and lymph node or distant metastases with the final impairment of the function of the sodium-iodide symporter and of other genes metabolising iodide, which can justify a more aggressive surgical approach (e.g. total thyroidectomy instead of lobectomy or frozen section) in patients with BRAF expression independently from the cytological category $(32,35,36,37,38,39,40,41,42,43)$. 
All this literature concerning not only the diagnostic role of BRAF but its prognostic correlation with aggressiveness is of high impact in the SM group, for which a more extensive surgical approach can be considered when BRAF mutated cases underwent surgery, avoiding frozen section or useless lobectomy.

Our 15 SM-BRAF mutated cases define a 100\% histological correlation with a diagnosis of thyroid carcinoma $(P=0.0353)$ and we are in agreement with the low incidence of BRAF mutation in the FVPC histotypes $(10 \%)$. In agreement with the XING report, our prognostic significance of BRAF was linked with $39 \%$ multifocal cancers and 32\% extra-thyroidal and nodal-positive cases $(37,43)$.

The same diagnostic and prognostic association of $B R A F$ expression was pointed out in the "positive for malignancy' category with the same significant correlation $(P=0.0009)$ with papillary cancer histotype and a clear correlation between BRAF expression and all the three aggressive parameters as lymph node metastases $(P<0.0001)$, extra-capsular invasion $(P=0.03)$ and multifocality $(P=0.0003)$.

To our knowledge, this is the first study aimed at the introduction of a preoperative cytological approach with the sequential application of both ICC and BRAF molecular tests in the category of SM on LBC. The positivity of ICC should be supported at least by two immunomarkers and although it has good $86.6 \%$ sensitivity and acceptable $63.6 \%$ negative predictive value, a collegial decision concerning the clinical management of these patients is advisable. Eventually, in our setting, patients with positive $B R A F$ evidence, based on the high $(100 \%)$ specificity of this technique and independently from the category to which they belong to, should be given total thyroidectomies with neck dissection, meaning a consequent reduction in the incidence of second surgery or intra-operative pathology in more than $40 \%$ of cases, and including specific prognostic and predictive information as well as selecting possible future specific targeting therapies $(30,43,44)$.

\section{Declaration of interest}

The authors declare that there is no conflict of interest that could be perceived as prejudicing the impartiality of the research reported.

\section{Funding}

This research did not receive any specific grant from any funding agency in the public, commercial or not-for-profit sector.

\section{References}

1 Fadda G \& Rossi ED. Liquid based cytology in fine-needle aspiration biopsies of the thyroid gland. Acta Cytologica 201155 389-400. (doi:10.1159/000329029)

2 Rossi ED, Raffaelli M, Minimo C, Mule A, Lombardi CP, Vecchio FM \& Fadda G. Immunocytochemical evaluation of thyroid neoplasms on thin-layer smears from fine-needle aspiration biopsies. Cancer 2005105 87-95. (doi:10.1002/cncr.21026)
3 Cochand-Priollet B, Prat JJ, Polivka M, Thienpont L, Dahan H, Wassef M \& Guillausseau PJ. Thyroid fine needle aspiration: the morphological features on ThinPrep slide preparations. Eighty cases with histological control. Cytopathology 200314 343-349. (doi:10.1046/j.0956-5507.2003.00098.x)

4 Fadda G, Basolo F, Bondi A, Bussolati G, Crescenzi A, Nappi O, Nardi F, Papotti M, Taddei G, Palombini L et al. Cytological classification of thyroid nodules. Proposal of the SIAPEC-IAP Italian consensus working group. Pathologica 2010102 405-408.

5 Cochand-Priollet B, Dahan H, Laloi-Michelin M, Polivka M, Saada M, Herman P, Guillausseau PJ, Hamzi L, Poté N, Sarfati E et al. Immunocytochemistry with cytokeratin 19 and HBME-1 increases the diagnostic accuracy of thyroid fine-needle aspirations. Preliminary report of 150 liquid-based fine needle aspirations with histological control. Thyroid 201121 1067-1073. (doi:10.1089/ thy.2011.0014)

6 Cheung CC, Ezzat S, Freeman JL, Rosen IB \& Asa SL. Immunohistochemical diagnosis of papillary thyroid carcinoma. Modern Pathology 200114 338-342. (doi:10.1038/modpathol. 3880312)

7 Herrmann ME, LiVolsi VA, Pasha TL, Roberts SA, Wojcik EM \& Baloch ZW. Immunohistochemical expression of Galectin-3 in benign and malignant thyroid lesions. Archives of Pathology $\mathcal{E}$ Laboratory Medicine 2002126 710-713.

8 Dabbs D, Abendroth CS, Grenko RT, Wang X \& Gail ER. Immunocytochemistry on the Thin-Prep Processor. Diagnostic Cytopathology 199717 388-392. (doi:10.1002/(SICI)10970339(199711)17:5<388::AID-DC14>3.0.CO;2-J)

9 Nikiforova MN \& Nikiforov Y. Molecular diagnostics and predictors in thyroid cancer. Thyroid 200919 1351-1361. (doi:10.1089/ thy.2009.0240)

10 Nikiforov YE, Steward DL, Robinson-Smith TM, Haugen BR, Klopper JP, Zhu Z, Fagin JA, Falciglia M, Weber K \& Nikiforova MN. Molecular testing for mutations in improving the fine needle aspiration diagnosis of thyroid nodules. Journal of Clinical Endocrinology and Metabolism $2009942092-2098$. (doi:10.1210/jc.2009-0247)

11 Soares P, Trovisco V, Rocha AS, Lima J, Castro P, Preto A, Máximo V, Botelho T, Seruca R \& Sobrinho-Simões M. BRAF mutations and RET/PTC rearrangements are alternative events in the ethiopathogenesis of PTC. Oncogene 200322 4578-4580. (doi:10.1038/sj.onc.1206706)

12 Cheung CC, Carydis B, Ezzat S, Bedard YC \& Asa SL. Analysis of RET/PTC gene rearrangements refines the fine needle aspiration diagnosis of thyroid cancer. Journal of Clinical Endocrinology and Metabolism 200186 2187-2190. (doi:10.1210/jc.86.5.2187)

13 Puxeddu E, Durante C, Avenia N, Filetti S \& Russo D. Clinical implications of BRAF mutation in thyroid carcinoma. Trends in Endocrinology and Metabolism 200819 138-145. (doi:10.1016/j. tem.2007.12.003)

14 Ohori NP, Nikiforova MN, Schoedel KE, LeBeau SO, Hodak SP, Seethala RR, Carty SE, Ogilvie JB, Yip L \& Nikiforov YE. Contribution of molecular testing to thyroid fine needle aspiration cytology of "follicular lesion of undetermined significance/atypia of undetermined significance". Cancer Cytopathology $2010 \mathbf{1 1 8}$ 17-23. (doi:10.1002/cncy.20063)

15 Tulecke MA \& Wang HH. ThinPrep for cytologic evaluation of follicular thyroid lesions: correlation with histologic findings. Diagnostic Cytopathology 200330 7-13. (doi:10.1002/dc.10391)

16 Scurry JP \& Duggan MA. Thin layer compared to direct smear in thyroid fine-needle aspiration. Cytopathology 200011 104-115. (doi:10.1046/j.1365-2303.2000.00228.x)

17 Afify AM, Liu J \& Al-Khafaji BM. Cytologic artefacts and pitfalls of thyroid fine needle aspiration using Thin Prep. Cancer Cytopathology 200193 179-186.

18 Malle D, Valeri RM, Pazaitou-Panajiotou K, Kiziridou A, Vainas I \& Destouni C. Use of thin layer technique in thyroid fine needle aspiration. Acta Cytologica $2006 \quad 50$ 23-27. (doi:10.1159/ 000325890) 
19 Ravetto C, Colombo L \& Dottorini ME. Usefulness of fineneedle aspiration in the diagnosis of thyroid carcinomas. A retrospective study in 37,895 patients. Cancer Cytopathology 2000 90 357-363.

20 Poller DN, Ibrahim AK, Cummings MH, Mikel JJ, Boote D \& Perry M. Fine-needle aspiration of the thyroid. Importance of an indeterminate diagnostic category. Cancer Cytopathology 200090 239-244.

21 Baloch ZW, LiVolsi VA, Asa SL, Rosai J, Merino MJ, Randolph G, Vielh P, DeMay RM, Sidawy MK \& Frable WJ. Diagnostic terminology and morphologic criteria for cytologic diagnosis of thyroid lesions: a synopsis of the National Cancer Institute fine-needle aspiration state-of-science conference. Diagnostic Cytopathology 200836 425-437. (doi:10.1002/dc.20830)

22 Fadda G, Rossi ED, Raffaelli M, Mulè A, Pontecorvi A, Miraglia A, Lombardi CP \& Vecchio FM. Fine-needle aspiration biopsy of thyroid lesions processed by thin-layer cytology: one-year institutional experience with histologic correlation. Thyroid $2006 \mathbf{1 6}$ 975-981. (doi:10.1089/thy.2006.16.975)

23 Saleh HA, Hammoud J, Zakaria R \& Khan AZ. Comparison of Thin-Prep and cell block preparation for the evaluation of thyroid epithelial lesions on fine needle aspiration biopsy. CytoJournal 20085 3. (doi:10.1186/1742-6413-5-3)

24 British Thyroid Association, Royal College of Physicians. Guidelines for the management of thyroid cancer Ed. P Perros 2nd edition. Report of the Thyroid Cancer Guidelines Update Group. London: Royal College of Physicians, 2007.

25 Cibas ES \& Ali SZ. The Bethesda system for reporting thyroid cytopathology. American Journal of Clinical Pathology 2009132 658-665. (doi:10.1309/AJCPPHLWMI3JV4LA)

26 Martini M, Teofili L, Cenci T, Giona F, Torti L, Rea M, Foà R, Leone G \& Larocca LM. A novel heterozygous HIF2AM535I mutation reinforces the role of oxygen sensing pathway disturbances in the pathogenesis of familial erythrocytosis. Haematologia 200893 1068-1071. (doi:10.3324/haematol.13210)

27 Faquin WC \& Baloch ZW. Fine needle aspiration of follicular patterned lesions of the thyroid. Diagnosis, management and follow-up according to national cancer institute (NCI) recommendations. Diagnostic Cytopathology 2010 38 731-739. (doi:10. 1002/dc.21292)

28 Mahajan A, Lin X \& Nayar R. Thyroid Bethesda reporting category, suspicious for papillary thyroid carcinoma, pitfalls and clues to optimize the use of this category. Cytopathology $2013 \mathbf{2 4}$ 85-91. (doi:10.1111/j.1365-2303.2012.00966.x)

29 Fadda G, Rossi ED, Raffaelli M, Pontecorvi A, Sioletic S, Morassi F, Lombardi CP, Zannoni GF \& Rindi G. Follicular thyroid neoplasms can be classified as low and high risk according to HBME-1 and Galectine 3 expression on liquid based fine needle cytology. European Journal of Endocrinology 2011165 447-453. (doi:10.1530/EJE-11-0181)

30 Correia-Rodrigues HG, Nogueira De Pontes AA \& Adan LFF. Use of molecular markers in samples obtained from preoperative aspiration of thyroid. Endocrine Journal $2012 \mathbf{5 9} 417-424$. (doi:10.1507/endocrj.EJ11-0410)

31 Chang H, Lee H, Yoon SO, Kim H, Kim A \& Kim BH. BRAF (V600E) mutation analysis of liquid-based preparation-processed fine needle aspiration sample improves the diagnostic rate of papillary thyroid carcinoma. Human Pathology 201243 89-95. (doi:10.1016/j.humpath.2011.04.010)

32 Mathur A, Weng J \& Moses W. A prospective study evaluating the accuracy of using combined clinical factors and candidate diagnostic markers to refine the accuracy of thyroid fine needle aspiration biopsies. Surgery $2010 \mathbf{1 4 8} 1176-1177$. (doi:10.1016/j.surg.2010.09.025)

33 Niemeier LA, Kuffner Akatsu H, Song C, Carty SE, Hodak SP, Yip L, Ferris RL, Tseng GC, Seethala RR, Lebeau SO et al.
A combined molecular-pathologic score improves risk stratification of papillary micro carcinoma. Cancer $2011 \mathbf{1 1 8}$ 2069-2077. (doi:10.1002/cncr.26425)

34 American Thyroid Association (ATA) Guidelines Taskforce on Thyroid Nodules and Differentiated Thyroid Cancer, Cooper DS, Doherty GM, Haugen BR, Kloos RT, Lee SL, Mandel SJ, Mazzaferri EL, McIver B, Pacini F et al. Revised management guidelines for patients with thyroid nodules and differentiated thyroid cancer. Thyroid 200919 1167-1214. (doi:10.1089/thy. 2009.0110)

35 Yip L, Farris C, Kabaker AS, Hodak SP, Nikiforova MN, McCoy KL, Stang MT, Smith KJ, Nikiforov YE \& Carty SE. Cost impact of molecular testing for indeterminate thyroid nodule fine-needle aspiration biopsies. Journal of Clinical Endocrinology and Metabolism 201207 1905-1912. (doi:10.1210/jc.2011-3048)

36 Cisco RM, Shen WT \& Gosnell JE. Extent of surgery for papillary thyroid cancer: preoperative imaging and role of prophylactic and therapeutic neck dissection. Current Treatment Options in Oncology 201013 1-10. (doi:10.1007/s11864-011-0175-z)

37 Xing M. Braf mutation in papillary thyroid cancer: pathogenic role, molecular bases, and clinical implications. Endocrine Reviews 200728 742-762. (doi:10.1210/er.2007-0007)

38 Oler G, Camacho CP, Hojaij FC, Michaluart P Jr, Riggins GJ \& Cerutti JM. Gene expression profiling of papillary thyroid carcinoma identifies transcripts correlated with BRAF mutational status and lymph node metastasis. Clinical Cancer Research 2008 14 4735-4742. (doi:10.1158/1078-0432.CCR-07-4372)

39 Musholt TJ, Fottner C, Weber M, Eichhorn W, Pohlenz J, Musholt PB, Springer E \& Schad A. Detection of papillary carcinoma by analysis of BRAF and RET/PTC1 mutations in fine needle aspiration biopsies of thyroid nodules. World Journal of Surgery 201034 2595-2603. (doi:10.1007/s00268-010-0729-4)

40 Park SY, Park YJ, Lee YJ, Lee HS, Choi SH, Choe G, Jang HC, Park SH, Park do J \& Cho BY. Analysis of differential BRAF (V600E) mutational status in multifocal papillary thyroid carcinoma. Evidence of independent clonal origin in distinct tumor foci. Cancer $2006 \mathbf{1 0 7} 1831-1838$. (doi:10.1002/cncr. 22218)

41 Eszlinger M \& Paschke R. Molecular fine-needle aspiration biopsy diagnosis of thyroid nodules by tumor specific mutations and gene expression patterns. Journal of Molecular and Cellular Endocrinology 2010322 29-37. (doi:10.1016/j.mce.2010.01.010)

42 Moses W, Weng J, Sansano I, Peng M, Khanafshar E, Ljung BM, Duh QY, Clark OH \& Kebebew E. Molecular testing for somatic mutations improves the accuracy of thyroid fine needle aspiration biopsy. World Journal of Surgery $2010342589-2594$. (doi:10.1007/s00268-010-0720-0)

43 Rossi ED, Martini M, Capodimonti S, Lombardi CP, Pontecorvi A, Vellone VG, Zannoni GF, Larocca LM \& Fadda G. BRAF (V600E) mutation analysis on LBC-processed aspiration biopsies predicts bilaterality and nodal involvement in papillary thyroid microcarcinoma. Cancer Cytopathology 2013. In press. (doi:10. 1002/cncy.21258)

44 Schmitt F. Molecular cytopathology and flow cytometry: preanalytical procedures matter. Cytopathology 201122 355-357. (doi:10.1111/j.1365-2303.2011.00941.x)

45 Schmitt FC, Longatto-Filho A, Valent A \& Vielh P. Molecular techniques in cytopathology practice. Journal of Clinical Pathology 200861 258-267. (doi:10.1136/jcp.2006.044347)

Received 8 January 2013

Revised version received 12 March 2013

Accepted 19 March 2013 